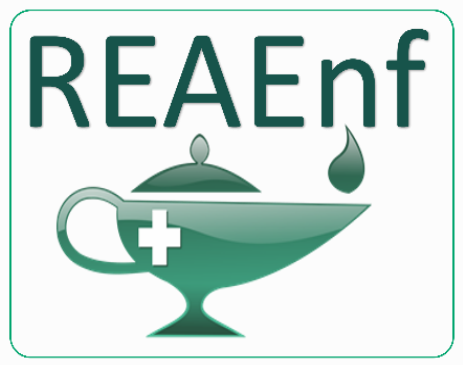

Revista Eletrônica Acervo Enfermagem
REVISÃO BIBLIOGRÁFICA

Recebido em: 1/2021

Aceito em: 2/2021

Publicado em: 3/2021

\title{
A Síndrome de Burnout e suas particularidades em profissionais de enfermagem no serviço de pronto atendimento emergencial
}

\author{
Burnout Syndrome and its particularities in nursing professionals in emergency service \\ El Syndrome de Burnout y sus particularidade en los profesionales de enfermería del \\ servicio de urgencias
}

Rosimeire da Silva ${ }^{1,2 *}$, Izabel Luiza dos Anjos Muniz ${ }^{2}$, Sadrake Tarsis Porto ${ }^{2}$, Viviane Araujo Cardoso?.

\begin{abstract}
Resumo: O objetivo desse trabalho foi reunir e revisar as possíveis reações psicoemotivas que permeiam as equipes de enfermagem e os fatores que desencadeiam a Síndrome de Burnout, principalmente no setor emergencial, pois estes profissionais estão constantemente em contato com elevados níveis de pressão e exigências do setor, estando propensos a apresentarem quadros de desgaste emocional consideravelmente mais intensos, o que pode levar à saturação do estado psicológico. Por meio dessa revisão narrativa, foi possível evidenciar as causas e as susceptibilidades trabalhistas específicas que predispõem ao surgimento desta patologia nos profissionais de enfermagem, assim como sugerir potenciais métodos de intervenção para reduzir os níveis de estresse ocupacional e, consequentemente, prevenir o desenvolvimento do distúrbio. Portanto, a Síndrome de Burnout é frequente e acomete muitos profissionais no ramo da enfermagem, no entanto essa ocorrência ainda é subestimada no Brasil. Diante disso, faz-se necessária a implementação de um diagnóstico efetivo do quadro patológico, para que a partir disso sejam implementadas medidas eficazes de prevenção e cuidados à saúde dos trabalhadores.
\end{abstract}

Palavras-chave: Enfermagem, Síndrome de Burnout, Trabalho.

\begin{abstract}
The aim of this work was to assemble related psycho-emotional reactions and causes related to nursing teams and the occurrence of Burnout Syndrome, especially in the emergency sector, because these professionals are constantly susceptible to high levels of pressure and demands, presenting more intense emotional exhaustion, which can lead to saturation of the psychological state. Through this narrative review, it was possible to highlight the causes and specific labor susceptibilities that predisposes the emergence of this pathology in nursing professionals, as well as to suggest potential intervention methods to reduce occupational stress levels and, consequently, prevent the development of this disorder. Therefore, Burnout Syndrome is frequent and affects many nurses, however this occurrence is still underestimated in Brazil. Thus, it is necessary to implement an effective diagnosis method of the pathological condition, to propose effective measures for prevention and health care of workers.
\end{abstract}

Key words: Nursing, Burnout Syndrome, Job.

Resumen: El objetivo de este trabajo fue recoger posibles reacciones psicoemocionales que permean los equipos de enfermería y los factores desencadenantes del Síndrome de Burnout, especialmente en sector de

${ }^{1}$ Centro Paula Souza (CPS), Jales - SP.

*E-mail: roosimeiresilva@hotmail.com

${ }^{2}$ Centro Universitário de Jales (UNIJALES), Jales - SP. 
emergencias, ya que estos profesionales están constantemente en contacto con altos niveles de presión y exigencias del sector, siendo propensos a presentar un agotamiento emocional considerablemente más intenso, que puede llevar a saturación del estado psicológico. A través de esta revisión narrativa se pudo resaltar las causas y susceptibilidades laborales que predisponen la aparición de esta patología en profesionales de enfermería, así como sugerir posibles métodos de intervención para reducir los niveles de estrés ocupacional y, en consecuencia, prevenir el desarrollo de la enfermedad desorden. Por lo tanto el Síndrome de Burnout es frecuente y afecta a muchos profesionales de enfermería, sin embargo, esta ocurrencia aún es subestimada en Brasil. Ante esto, es necesario implementar un diagnóstico efectivo de la condición patológica, para que implementen medidas efectivas en prevención y atención de la salud de los trabajadores.

Palabras clave: Enfermería, Síndrome de Burnout, Trabajo.

\section{INTRODUÇÃO}

Entre os profissionais da saúde, a equipe de enfermagem está constante e diretamente em contato com os pacientes e familiares no setor emergencial, de tal maneira que a prática assistencial ultrapassa os limites profissionais, atingindo também as esferas emocionais e afetivas para com os pacientes (GIANASI LBS e OLIVEIRA DC, 2014).

A enfermagem é vista como a mais antiga entre as práticas de assistência à saúde, pois denota desde a época pré-cristã, perpassando por diversos períodos históricos, conforme o conhecimento a respeito do tratamento de doenças e acometimentos foi sendo acumulado ao longo dos anos (MORAIS SCRV, et al., 2010).

Avaliada como uma das profissões mais desgastantes, a enfermagem enfrenta inúmeros desafios, tais como: baixo salário; falta de reconhecimento profissional; carga horária excessiva; escassez de pessoal, que culmina no acúmulo de tarefas e na sobrecarga laboral; exposição constante ao sofrimento do paciente; condições de trabalhos precárias; baixa participação nas escolhas e decisões no ambiente de trabalho; problemas interpessoais decorrentes da convivência, entre outros que podem eventualmente contribuir para o desenvolvimento da chamada Síndrome de Burnout (DIAS FM, et al., 2016).

O termo "Burnout", originário do inglês ("burn out"), pode ser traduzido como "queima após o desgaste" ou "esgotamento", sendo o seu significado associado justamente a um processo de desgaste e esgotamento profissional desenvolvido no ambiente de trabalho, cujas características possuem relação com aspectos pessoais do indivíduo e se projetam em três pilares principais: o cansaço emocional; a impessoalização e a frustração (ou irrealização) profissional (MOTA BS, et al., 2020; PIRES FC, et al., 2020).

Este distúrbio apresenta especificidades sintomatológicas no âmbito do trabalho em enfermagem, as quais não podem ser confundidas com "simples estresse", uma vez que o estresse é uma agressão que perturba o equilíbrio interno dos seres humanos, enquanto a Síndrome de Burnout é a resposta fisiopatológica ao estresse laboral crônico (erroneamente interpretado e confundido como estresse comum no ambiente de trabalho, principalmente quando o trabalhador não consegue atender às demandas solicitadas pela empresa), ocasionando atitudes e alterações comportamentais negativas no contexto ocupacional (DIAS FM, et al., 2016).

Nesse sentido, ao observarmos as especificidades da síndrome e o contexto de trabalho dos enfermeiros, é razoável supor que estes profissionais da saúde provavelmente são mais susceptíveis a desenvolverem tal distúrbio psíquico, uma vez que os muitos desafios da profissão (cuidado constante com os pacientes, imprevisibilidade, rotina diária, baixa remuneração, sobrecarga de turnos, entre outros) são frequentes e comuns nas unidades de saúde e setores emergenciais do país (PIRES FC, et al., 2020).

Portanto, com base no grau de importância e relevância do tema, o presente artigo objetivou a realização de um levantamento bibliográfico para construção de uma revisão narrativa, a fim de compilar estudos científicos da área e abordar os principais aspectos relacionados às causas que predispõem os profissionais de enfermagem ao desenvolvimento da Síndrome de Burnout. 


\section{REVISÃO BIBLIOGRÁFICA}

A realidade em saúde no Brasil apresenta precariedade em determinadas regiões, principalmente quando nos referimos às estruturas básicas de "gestão de saneamento", as quais são capazes de evidenciar especificamente o tratamento biológico do contexto saúde-doença, que impacta diretamente nos cuidados e na qualidade de saúde dos pacientes. Isso significa que a política de ação em saúde no nosso país necessita ser revista, pois a sociedade brasileira culturalmente não tem dimensão da conjuntura dos processos relacionados à área da saúde (FERREIRA PSF, et al., 2016).

A enfermagem, que atua diretamente no processo assistencial da saúde, deve utilizar-se de um conjunto de ações, definidas por habilidades profissionais e inúmeros conhecimentos específicos, a fim de agir e oferecer um cuidado integral aos pacientes, proporcionando concomitantemente equilíbrio e energia também aos funcionários, os quais contribuem como importantes fatores de integridade do sistema funcional como um todo (SILVA JF, et al., 2020).

Nesse sentido, tem-se então uma área de atuação composta por profissionais que podem ser considerados o anteparo da realidade salutar, estando diretamente relacionados ao cuidado e à manutenção do estado clínico de terceiros e, portanto, requerendo certo poder de articulação para desempenharem suas funções com eficiência e assertividade (OJEDA BS, et al., 2014).

A formação do profissional de enfermagem passou a ter um novo perfil, sendo preconizada pela Lei de Diretrizes e Bases no 9.394/96, a qual tem sido amplamente discutida pelas instituições formadoras de ensino superior. As novas diretrizes e regulamentos destinados à atuação profissional dos enfermeiros evidenciam a necessidade de os trabalhadores da área desenvolverem ao longo de sua formação pilares como: qualidade técnica, conhecimentos básicos de caráter global, capacidade de tomada de decisões e de empreendimento, senso de colaboração para trabalho em equipe e desenvolvimento de habilidades para lidar com a imprevisibilidade constante do ambiente trabalho (SOUZA SNDH e MIYADAHIA AMK, 2012).

Na maioria das vezes, quando nos referimos à saúde mental fazemos menção à ausência de transtornos mentais, deficiências, ou anomalias genéticas diagnosticadas, mas, segundo a Organização Mundial de Saúde (OMS), a saúde mental pode ser perturbada por inúmeros fatores (sociais, biológicos, econômicos ou ambientais), sendo então um fator determinante para a saúde dos indivíduos como um todo (BRASIL, 2018).

Estudos justificam que dentre as diversas categorias profissionais inseridas no setor da saúde, os profissionais de enfermagem são os que apresentam maiores índices de acidentes ocupacionais e agravos à saúde mental. Sendo assim, no contexto profissional da enfermagem, a organização do trabalho compreende questões que são relevantes para a saúde ocupacional, as quais estão relacionadas aos seguintes fatores: à sua divisão, hierarquia, regras formais, produtividade, jornada de trabalho, natureza da atividade, tempo para pausa e descanso, entre outros (RODRIGUES CCFM e SANTOS VEP, 2017).

O setor de urgência e emergência é diário e ininterrupto, pois oferece a possibilidade de atendimento 24 horas por dia e tem como público-alvo os indivíduos que necessitam de cuidados imediatos e/ou que apresentem risco de morte. Consequentemente, os profissionais que atendem nessas unidades de saúde devem ser/estar preparados para realizar procedimentos que ofereçam condições adequadas para o exercício profissional consciente, competente e seguro, tanto aos próprios prestadores de serviços, como também aos pacientes atendidos (PIRES FC, et al., 2020).

Diante de tais circunstâncias, o serviço de urgência e emergência se desenrola diariamente em uma atmosfera complexa e desafiadora, cujas demandas exigem a contratação de profissionais aptos, competentes e preparados, tanto intelectual, como psicologicamente, para que seja possível proporcionar um atendimento imediato, adequado, e com padrão elevado de excelência, tal qual a natureza do serviço exige (CRUZ FMP, et al., 2020).

Considerando o elevado nível de pressão e exigências do setor, torna-se importante mencionar e destacar que os profissionais de enfermagem que atuam nas unidades de emergência estão propensos a apresentarem quadros de desgaste emocional consideravelmente mais intensos, pois a sobrecarga de 
trabalho vem aumentando diária e progressivamente, levando-os à saturação do estado psicológico e mostrando-nos a importância de considerar as diversas variáveis estressoras do ambiente de trabalho (CRUZ FMP, et al., 2020; PORTELA NLC, et al., 2015).

As particularidades no âmbito da saúde dos enfermeiros estão relacionadas a relatos frequentes de dores, alergias, alteração da pressão arterial, mal-estar orgânico, insônia, sofrimento emocional e físico. Isso significa, em outras palavras, que os profissionais dessa área apresentam uma carga adicional de competências e responsabilidades, originária de pressões no ambiente de trabalho, que pode culminar em um estado de estresse crônico (CRUZ FMP, et al., 2020; SIMÕES J, 2020).

A Síndrome de Burnout foi recentemente inclusa na 11를 Classificação Internacional de Doenças (CID-11) no capítulo "Fatores que influenciam o estado de saúde ou o contato com serviços de saúde", sendo classificada apenas como um fenômeno ocupacional, sem ser relacionada ou associada a um quadro patológico específico (MOTA BS, et al.,2020; WHO, 2019).

Neste mesmo documento, a síndrome é descrita como sendo resultante do estresse crônico laboral, cuja administração não ocorreu de maneira bem-sucedida ou não the deram a devida atenção, podendo ser caracterizada por três pilares principais: 1) sensação de esgotamento ou exaustão de energia (especificamente no contexto ocupacional); 2) distanciamento mental, sentimentos negativos ou cinismo relacionado ao trabalho; e 3) eficácia profissional reduzida (ANDRADE FM, et al., 2019; WHO, 2019).

Os fatores de risco, na maioria dos casos, estão associados tanto à ocorrência de acidentes de trabalho, como também ao esgotamento emocional, que muitas vezes resultam em descontrole com colegas ou até mesmo com pacientes, além de crises de choro, dores abdominais intensas, crises de pânico, entre outras situações que podem resultar em consequências incômodas para o próprio profissional, para o paciente e também para o gestor da empresa (BARROSO AL, et.al., 2020).

O processo para diagnóstico da Síndrome de Burnout conta com a avaliação e associação de diversos sintomas, que nas suas especificidades podem ser divididos em: 1) sintomas físicos: distúrbios do sono, enxaquecas, fadiga constante, disfunções sexuais, entre outros; 2 ) sintomas psíquicos: falta de atenção, falta de concentração, lentidão de pensamento, solidão, desânimo, entre outros; 3) sintomas comportamentais: negligência, irritabilidade, dificuldade na aceitação de mudanças, comportamento de alto risco, e outros; e 4) sintomas defensivos: isolamento, perda de interesse pelo trabalho, ironia, ímpetos de abandono do trabalho, entre outros (BRAYDE DHS e COSTA TNM, 2019; BENEVIDES-PEREIRA AMT, 2002; MONTENEGRO LC, 2010).

Embora se conheça a grande associação mantida entre os sintomas físicos e psicológicos em casos de estresse, alguns estudos relatam que as manifestações físicas são mais comumente reportadas do que as emocionais, isso porque, normalmente, as pessoas apresentam maior resistência em admitir sintomas de estresse psicológico, uma resposta cultural às demandas ambientais e sociais da comunidade, que exigem cada vez mais a manutenção do controle da situação e de uma vida estável. Apesar dessa resistência, o estresse é citado com frequência e de forma casual pelos trabalhadores, quase como um fenômeno natural (UENO LGS, et al., 2017).

O estresse em si pode ser considerado um sintoma, mas também uma das principais causas da Síndrome de Burnout (MACIEL APN e GONÇALVES JR, 2020). Nesse sentido, diversos estudos colocam o estresse como consequência de políticas inadequadas da instituição de saúde quanto ao ambiente que compõe a atmosfera de trabalho. Essas tensões levam ao aparecimento frequente de atritos entre os funcionários, administradores e outros profissionais, fazendo com que a competitividade interna, o sentimento de inveja e a falta de habilidade nas relações interpessoais sejam intercorrências frequentemente observadas no setor (RIBEIRO RP, et al., 2018).

Isso evidencia que os profissionais de saúde (enfermeiros, médicos, bombeiros, socorristas de pronto atendimento emergencial, entre outros) ou de instituições que apresentam uma estrutura organizacional de atendimento público com sobrecarga de trabalho, como por exemplo o setor da educação, deveriam ser submetidos periodicamente a avaliações psicométricas, mas devido à falta de conhecimento mais 
aprofundado a respeito da Síndrome de Burnout, muitas vezes esse distúrbio psíquico é enquadrado como estresse "comum" ou transtorno depressivo, e não como uma condição crônica ocupacional com características tais que demandem certa atenção especial (LIMA CF, et al., 2009).

Torna-se importante destacar que na esfera da assistência trabalhista há uma relação conflitante por parte da organização estrutural e os profissionais de enfermagem que são pré-diagnosticados com estresse ocupacional, pois a avaliação, que é individualizada, muitas vezes apresenta caracteres e critérios errôneos, não permitindo a confirmação da Síndrome de Burnout, mas sim de outras enfermidades como, por exemplo, transtornos depressivos, de ansiedade, de pânico, ou outros (VASCONCELOS EM e MARTINO MMF, 2017).

Nesse sentido, apesar dos números atuais relacionados às ocorrências de transtornos mentais e doenças físicas em profissionais da saúde serem elevados, o reconhecimento e diagnóstico clínico da Síndrome de Burnout em si pode ser considerado baixo, alcançando no estado de São Paulo, por exemplo, a média de $14,3 \%$ (VASCONCELOS EM e MARTINO MMF, 2017). Por outro lado, pesquisas realizadas pela International Stree Management (ISMA) incluem o Brasil como o segundo país com maior porcentagem populacional de ocorrência da síndrome (70\%), estando apenas atrás do Japão (SILVA JF, et al., 2020).

Observa-se que muitos dos profissionais da enfermagem, expostos ao estresse ocupacional diariamente, têm apresentado inúmeras alterações em parâmetros fisiológicos, tais como a hiperglicemia, hiperlipidemia, alterações neuroendócrinas, e outras que podem desencadear riscos de desenvolver doenças cardiovasculares a longo prazo, por exemplo. Além disso, os relatos de uso abusivo de álcool e outras substâncias psicoativas têm sido frequentes, 0 que pode ocasionar e potencializar implicações socioeconômicas como o abandono do trabalho, queda de produtividade, descaso com a carreira, isolamento social, entre outros inúmeros fatores (LIMA AS, et al., 2018).

A atividade profissional deve, ou pelo menos deveria, ser fonte de satisfação no percurso pela busca do reconhecimento ocupacional e econômico, proporcionado ao trabalhador sua reafirmação enquanto pessoa, estimulando o desenvolvimento da sensação constante de procura por doar-se de forma saudável e cuidadosa ao trabalho, e contribuindo para a construção do bem-estar mental de terceiros e de si próprio (NOGUEIRA LS, et al., 2018).

Diante destes aspectos, políticas de enfrentamento e estratégias para prevenir a Síndrome de Burnout devem ser consideradas, valorizando a promoção dos valores humanos no ambiente de trabalho, sendo que, cada indivíduo deve realizar um processo de mudança pessoal e institucional, apresentando em conjunto, propostas construtivas e participativas para o bem-estar de todos no ambiente de trabalho, através de esforços cognitivos e comportamentais voltados para o manejo de demandas externas ou internas (PÊGO FPL e PÊGO DR, 2016).

Afim de destacar a importância da implementação de medidas preventivas, Perniciotti $P$, et al. (2020) evidenciaram uma tríade de intervenções principais para a prevenção da Síndrome de Burnout, sendo elas: individuais, organizacionais e/ou combinadas.

As intervenções individuais dizem respeito a modificações pessoais do sujeito, pautadas na aprendizagem de estratégias para a condução saudável de situações estressantes. Por outro lado as intervenções organizacionais versam sobre modificações no ambiente de trabalho, baseadas no diálogo entre os membros da equipe e na abertura para inclusão de medidas como: treinamento de funcionários; readequação de tarefas e funções; alterações nas condições físico-ambientais do local de trabalho; flexibilização da carga horária; possibilidade de plano de carreira e autonomia laboral. Finalmente, as medidas interventivas combinadas são caracterizadas pela ação conjunta das intervenções individuais e organizacionais, sendo as mais adequadas e recomendadas, pois permitem a modificação da atmosfera de trabalho como um todo, contribuindo com a redução dos níveis de estresse ocupacional e, consequentemente, prevenindo o desenvolvimento da Síndrome de Burnout (PERNICIOTTI P, et al., 2020).

Nesse contexto vale salientar que os riscos do referido distúrbio são únicos para cada indivíduo e possuem um grande espectro de possibilidades sintomatológicas, assim, a confirmação do diagnóstico deve ser analisada e avaliada com cautela pelos profissionais responsáveis, após várias sessões de entrevistas clínicas e aplicação adequada de métodos de avaliação psicológica (BARBOSA THA, et al., 2021). 
Portanto, considerando que os profissionais do setor emergencial realizam esforços diariamente para prestar uma assistência rápida e de qualidade ao seu público-alvo e que é visível a susceptibilidade e desenvolvimento de quadros de estresse crônico nestes trabalhadores, fica evidente a importância de ser realizar o diagnóstico da Síndrome de Burnout de forma assertiva, com base na avaliação de inúmeros parâmetros e aspectos multifatoriais.

\section{CONSIDERAÇÕES FINAIS}

Com base no desconhecimento do nível de ocorrência da Síndrome de Burnout no Brasil e seus respectivos impactos nos âmbitos social, salutar e econômico, faz-se necessária a reavaliação dos métodos diagnósticos do distúrbio especialmente para os profissionais da saúde, pois somente por meio da obtenção destes dados, será possível compreender a real proporção do problema e sugerir métodos eficazes que promovam um ambiente de trabalho saudável e seguro a todos. Por fim, sugere-se que sejam implementados métodos de intervenção pautados no cuidado à saúde dos enfermeiros, com práticas de relaxamento em grupo, programas de ginástica laboral, rodízio de setores e psicoterapia, a fim de minimizar a ocorrência da síndrome e, consequentemente, melhorar a qualidade da assistência e do serviço prestados.

\section{REFERÊNCIAS}

1. ANDRADE FM, et al. Síndrome de Burnout em profissionais de enfermagem no ambiente hospitalar: uma revisão integrativa. Revista Eletrônica Acervo Saúde, 2019; 20: 1-9.

2. BARBOSA THA, et al. A síndrome de burnout e suas consequências nos profissionais de saúde: uma revisão de literatura. Brazilian Journal of Health Review, 2021; 4: 37-39

3. BARROSO AL, et al. Síndrome de Burnout na enfermagem: fatores associados ao processo de trabalho. Reserarch, Society and Development, 2020; 9: 1-13.

4. BENEVIDES-PEREIRA AMT. Burnout: quando o trabalho ameaça o bem-estar do trabalhador. São Paulo: Caso do Psicólogo, 2002.

5. BRASIL. Secretaria de Estado da Saúde do Espírito Santo. Diretrizes Clínicas em Saúde Mental. 1ae ed., 2018.

6. BRAYDE DHS, COSTA TNM. Prevalência de Síndrome de Burnout em médicos residentes de um hospital de ensino. Revista Eletrônica Acervo Saúde, 2019; 24: 1-7.

7. CRUZ FMP, et al. Impactos decorrentes da síndrome de burnout nos profissionais do Serviço de Atendimento Móvel de Urgência (SAMU). Revista Eletrônica Acervo Saúde, 2020; 12(10): 1-11.

8. DIAS FM, et al. O estresse ocupacional e a síndrome do esgotamento profissional (burnout) em trabalhadores da indústria do petróleo: uma revisão sistemática. Revista Brasileira de Saúde Ocupacional, 2016; 41: 11-12.

9. FERREIRA PSF, et al. Avaliação preliminar dos efeitos da ineficiência dos serviços de saneamento na saúde pública brasileira. Revista Internacional de Ciências, 2016; 6: 214-229.

10. GIANASI LBS, OLIVEIRA DC. A síndrome de burnout e suas representações entre profissionais de saúde. Estudos e Pesquisas em Psicologia, 2014; 14(3): 756-772.

11. LIMA AS, et al. Análise da prevalência da Síndrome de Burnout em profissionais da atenção primária em saúde. Trabalho, Educação e Saúde, 2018; 16(1): 283-304.

12. LIMA CF, et al. Avaliação Psicométrica do Maslach Burnout Inventory em Profissionais de Enfermagem. II Encontro de Gestão de Pessoas e Relações de Trabalho (EnGPR), 2009.

13. MACIEL APN, GONÇALVES JR. Incidência da Síndrome de Burnout na enfermagem. Revista JRG de Estudos Acadêmicos, 2020; 3(6): 96-109.

14. MONTENEGRO LC. A formação profissional do enfermeiro: avanços e desafios para a sua atuação na atenção primária à saúde. Dissertação (Mestrado em Enfermagem e Saúde) - Universidade Federal de Minas Gerais, Belo Horizonte, 2010; 98 p.

15. MORAIS SCRV, et al. O cuidar em enfermagem à mulher vítima de violência sexual. Texto \& Contexto - Enfermagem, 2010; 19(1): 155-160.

16. MOTA BS, et al. As contribuições da síndrome de Burnout para o déficit do trabalho da enfermagem: revisão integrativa da literatura. Revista Eletrônica Acervo Saúde, 2020; 12(10): 1-10.

17. NOGUEIRA LS, et al. Burnout e ambiente de trabalho de enfermeiros em instituições públicas de saúde. Revista Brasileira de Enfermagem, 2018; 71(2): 358-365.

18. OJEDA BS, et al. A integração ensino e assistência na enfermagem: delineando possibilidades para uma prática contextualizada. Acta Paulista de Enfermagem, 2004; 17(4): 432-438.

19. PÊGO FPL, PÊGO DR. Síndrome de Burnout. Revista Brasileira de Medicina do Trabalho, 2016; 14(2): $171-176$.

20. PERNICIOTTI P, et al. Síndrome de Burnout nos profissionais de saúde: atualização sobre definições, fatores de risco e estratégias de prevenção, 2020; 23(1): 35-52.

21. PIRES FC, et al. Síndrome de Burnout em profissionais de enfermagem de pronto-socorro. Revista de Enfermagem - UFPE online, 2020; 14: 1-7. 
22. PORTELA NLC, et al. Síndrome de burnout em profissionais de enfermagem de serviços de urgência e emergência. Revista de Pesquisa Cuidado é fundamental Online, 2015; 72(3): 2749-2760.

23. RIBEIRO RP, et al. Estresse ocupacional entre trabalhadores de saúde de um hospital universitário. Revista Gaúcha de Enfermagem, 2018; 39: 1-6.

24. RODRIGUES CCFM, SANTOS VEP. Patient safety and nursing: interface with stress and Burnout Syndrome. Revista Brasileira de Enfermagem, 2017; 70(5): 1083-1088.

25. SILVA JF, et al. Síndrome de Burnout em profissionais de Enfermagem no contexto da Atenção Básica. Revista Eletrônica Acervo Saúde, 2020; 39: 1-7.

26. SIMÕES J. Síndrome de Burnout na equipe de enfermagem: desafios e perspectivas: uma revisão de literatura. Arquivos do Mudi, 2020; 24(1): 133-144.

27. SOUZA SNDH, MIYADAHIRA AMK. O desenvolvimento de competências no curso de graduação em enfermagem: percepção de egressos. Ciência, Cuidado e Saúde, 2012; 11(5): 243-250.

28. UENO LGS, et al. Estresse ocupacional: estressores referidos pela equipe de enfermagem. Revista de Enfermagem UFPE Online, 2017; 11(4): 1632-1638.

29. VASCONCELOS EM, MARTINO MMF. Preditores da síndrome de Burnout em enfermeiros de unidade de terapia intensiva. Revista Gaúcha de Enfermagem, 2017; 38(4): 1-8.

30. WHO. Suicide in the world: Global Health Estimates. World Health Organization, 2019. 